\title{
Magnetohydrodynamic Thin Film Flow through a Porous Stretching Sheet with the Impact of Thermal Radiation and Viscous Dissipation
}

\author{
Ziad Khan (D), Muhammad Jawad $\mathbb{1}$, ${ }^{1}$ Ebenezer Bonyah $\left(\mathbb{D},{ }^{2}\right.$ Naveed Khan $\left(\mathbb{D},{ }^{1}\right.$ \\ and Rashid Jan $\mathbb{B D}^{1}$ \\ ${ }^{1}$ Department of Mathematics, University of Swabi, Swabi 23561, Khyber Pakhtunkhwa, Pakistan \\ ${ }^{2}$ Department of Mathematics Education, Akenten Appiah Menka University of Skills Training and Entrepreneurial Development, \\ Kumasi, Ghana
}

Correspondence should be addressed to Ebenezer Bonyah; ebbonya@gmail.com

Received 16 September 2021; Revised 27 December 2021; Accepted 31 December 2021; Published 19 January 2022

Academic Editor: Shams -ul-Islam

Copyright (c) 2022 Ziad Khan et al. This is an open access article distributed under the Creative Commons Attribution License, which permits unrestricted use, distribution, and reproduction in any medium, provided the original work is properly cited.

In this paper, the Darcy-Forchheimer laminar thin film flow with MHD and heat transfer on an unsteady horizontal stretched surface is investigated. The impact of thermal radiation and viscous dissipation is also considered for thin film flow. The analysis of heat source with thermal radiation in a boundary layer flow can play a great role in manufacturing engineering procedures such as the production of electric power, solar energy modernization, and astrophysical flow. By using similarity transformation, the system of PDEs is converted to ODEs. Then HAM is applied for the solution of the problem. Moreover, the velocity and temperature profile for various embedded variables are discussed through graphs while the numerical solution of concerned physical quantities such as skin friction and Nusselt number are discussed through tables. The analysis shows that velocity profile is reducing function of $M, K_{1}, S$, and $F_{1}$. The temperature profile is an increasing function $S$ and $E c$ while a reducing function of Pr and $R$. The overall behavior of the proposed system is highlighted analytically.

\section{Introduction}

Heat source, fluid flow within liquid film due to an unsteady stretching sheet, plays a very crucial role in many utilization engineering processes and technologies; for example, polymer extrusion, continuous casting, manufacturing plastic film, tinning of copper wires, crystal growth, a polymer sheet, artificial fiber, and wire and fiber coating. In addition to these, many production processes involve heat transmission in various forms such as cooling of a machine tool, pasteurization of food, heat treatment of a part in industrial furnaces, or the temperature control for initiating a chemical reaction. Heat transfer is accomplished in most of these applications using heat transfer instruments such as heat swaps, desiccation, condensers, boilers, and heat sinks. Mathematics plays an important role in different areas of research and has effective outcomes [1-4]. The importance of mathematical tools in the investigation of fluid flow cannot be ignored. Mathematical tools help the researchers to visualize the dynamical behavior of a system and to conceptualize the importance of the input factors of a system. Numerous researchers workout to understand the flow of fluid with different assumptions and in different circumstances. The nanofluid flow over a stretching surface has been originally considered by Wang [5]; they also reduce unstable Navier-Stokes equations using similarity transformation in nonlinear ODEs, which are then calculated by multiple shooting methods. The solutions based on the analysis of a liquid thin film are obtained by Wang [6], who also validated the correctness of the homotopy analysis technique. Sufian et al [7] study the impact of heat source and fluid flow over a stretch cylinder as a function of time. Zhang et al. [8] demonstrated mass diffusion and solutal Marangoni impact on heat transmission and fluid flow in a 
film toward a stretched sheet. Kalyani et al. [9] investigated the effects of Lorentz force, heat generation, and mass transfer of a Casson incompressible fluid flowing on a surface. They also discussed the impact of thermal radiation on the mass and heat transfer property of flow in a Casson liquid film [10]. The unstable mixed convection flow of the stretch sheet is considered by Devi et al. [11]. Jhankal et al. [12] studied heat source, the forced convective flow of an incompressible fluid past plate inserted in a Darcy-Forchheimer porous medium of the two-dimensional boundary layer MHD flow. The effects of thermal radiation in an upper-convective Maxwell thin film, unsteady stretched sheet inserted in a porous medium are conceptualized by Waheed [13]. Ahmad et al. [14] illustrated a solution for the heat source, MHD flow toward an exponentially stretched surface in the absence of radiation impacts and Darcy's resistance. On the basis of boundary layer hypothesis and similarity transformation, Sparrow and Gregg [15] were the first to examine the Laminar-film condensing difficulty on a vertical plate. Dandapat and Ray explored the flow of a thin boundary layer across a spinning horizontal disc $[16,17]$. Nath and Kumari [18] investigated the time-dependent MHD problem of a fluid dispersed by a spinning incompressible disc. Magnetohydrodynamic and heat flow toward a stretch sheet has been studied by Mohd Nasir et al. [19] in which we consider both the stretching and shrinking cases. Zaidi et al. [20] studied the impacts of thermal radiation, heat creation, and induced magnetic field on hydromagnetic free convection flow of pair stress fluid in an isoflux-isothermal vertical channel. Kishore et al. [21] studied the influence of thermal radiation and viscous dissipation on MHD mass, heat transfer flow past an oscillating vertical plate inserted in a porous medium with unknown surface situations. Elbashbeshy et al. [22] studied the influence of thermal radiation and heat transmission on an unstable extended sheet inserted in a porous medium in the existence of a heat sink. Thermal radioactivity and heat transfer of Sisko fluid on an uneven stretched sheet with a constant magnetic field were investigated by Khan et al. [23]. The authors of [24] discussed mass transfer and unstable boundary layer MHD flow of a UCM fluid in the absence of higher-level chemical reaction. The authors of [25] considered heat transfer, MHD flow in a liquid film over an unsteady stretched sheet. Numerical solution of MHD boundary layer flow of a nanofluid over a moving sheet in the absence of thermal radiation has been carried out by Shateyi and Prakash [26]. The MHD laminar boundary layer flow with heat and mass transport of an electrically conducting water-based nanofluid across a nonlinear stretching sheet with viscous dissipation is studied by Mabood and Khan in [27]. It has been noticed that further investigation is needed to conceptualize the impact of heat transfer, thermal radiation in a fluid film of an unsteady stretching surface inserted in a porous medium in the absence of viscous dissipation and magnetic field impact. Therefore, in the current work, we opt to investigate the impact of heat transfer, thermal radiation in a fluid film of an unsteady stretching surface inserted in a porous medium in the absence of viscous dissipation and magnetic field impact.

The aim of the present work is to study Darcy-Forchheimer's laminar thin film flow on an unsteady horizontal stretched surface. The laminar thin-film flow is investigated with the impact of $\mathrm{MHD}$, thermal radiation, and viscous dissipation. The HAM is used to obtain analytic approximations for the solution of nonlinear ODEs. The comparison of HAM and numerical solutions are displayed. The results have been illustrated in graphical or tabular forms followed by a brief discussion and physical interpretations.

\section{Mathematical Expressions of the Problem}

2.1. Basic Equations and Boundary Constraints. Assume a thin liquid film extending on a stretched surface with a laminar unsteady flow which arises from a narrow slit where $\mathrm{x}$-coordinates are parallel to the slit and $y$-coordinates are perpendicular to the sheet as exhibited in Figure 1. Along $x$-axis, the stretching sheet is stretched by two equal and opposite forces. Further, the latent heat effect is neglected due to evaporation by assuming the liquid to be nonvolatile. Moreover, due to a relatively thin film, the buoyancy force is also neglected.

The continuous sheet velocity is given by $U(x, t)=c x /(1-\gamma t)$ [25], where $c$ and $\gamma$ are constants having per-time dimension. The term $c x^{2} /(\nu 1-\gamma t)$ is localized Reynolds number on the sheet velocity $U$, and the stretched rate $c /(1-\gamma t)$ reduces with time as $0 \leq \gamma<1$. The nomenclature of this research work has been presented.

The stretched sheet's surface temperature $T_{s}$ is determined as [25]

$$
T_{s}(x, t)=T_{0}-T_{r e f}\left[\frac{c x^{2}}{2 \nu}\right](1-\gamma t)^{-3 / 2},
$$

where $T_{0}$ is the slit temperature and $T_{\text {ref }}$ is the temperature with reference, which might be a constant temperature with a reference or a constant temperature difference. In this work, $T_{\text {ref }}$ will be interpreted as $0 \leq T_{\text {ref }} \leq T_{0}$. Normally, a transverse magnetic field is applied to the extended surface, which is believed to be changeable in nature and chosen as

$$
B(x, t)=B_{0}(1-\gamma t)^{-1 / 2} \text {. }
$$

The temperature and velocity influence of a thin film obeys the following governing time-dependent equations with the boundary conditions [25]. 


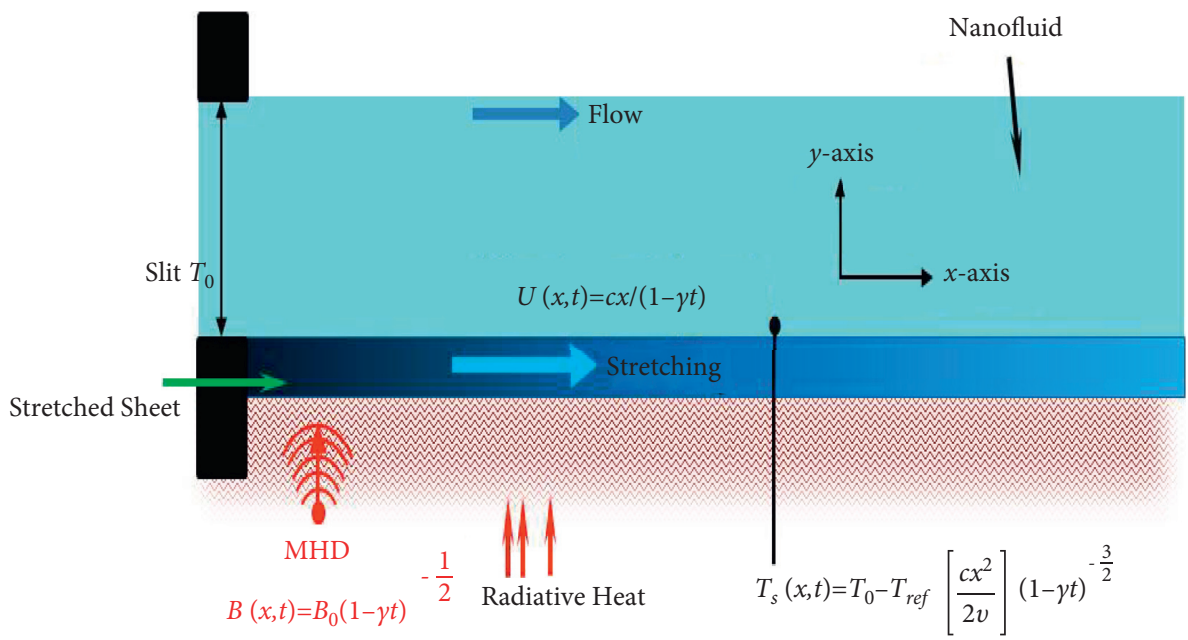

FIgURE 1: Geometric representation of thin film to illustrate its structure.

$$
\begin{aligned}
& \frac{\partial u}{\partial x}+\frac{\partial v}{\partial y}=0 \\
& \frac{\partial u}{\partial t}+u \frac{\partial u}{\partial x}+v \frac{\partial u}{\partial y}=v \frac{\partial^{2} u}{\partial y^{2}}-\frac{\sigma B_{0}^{2}}{\rho} u-\frac{\nu \varepsilon^{2}}{k} u-F u^{2} \\
& \frac{\partial T}{\partial t}+u \frac{\partial T}{\partial x}+v \frac{\partial T}{\partial y}=\frac{k}{\rho C_{p}} \frac{\partial^{2} T}{\partial y^{2}}+\frac{\mu}{\rho C_{p}}\left(\frac{\partial u}{\partial y}\right)^{2}+\frac{1}{\rho C_{p}} \frac{\partial q_{r}}{\partial y}
\end{aligned}
$$

where $u$ and $v$ denote the velocity terms in the directions of $x$ axis and $y$-axis, respectively, $\mu$ is the dynamic viscosity, $B_{0}$ is the magnetic field, $\rho$ is the density, $T$ is the temperature, $k$ is the thermal diffusivity, $v$ is the kinematic viscosity, $t$ is the time, $\sigma$ is the electrical conductivity, and $h$ is the uniform thickness of the film sheet. In the $\mathrm{x}$ - and $\mathrm{y}$-directions, the two terms of equation (3) reflect the net outflow (outflow minus inflow) of mass, for an unsteady flow the sum of these terms must be zero. The net rate at which $\mathrm{x}$-momentum leaves due to fluid motion across its limits is signified by the 2 nd and 3rd components on the left side of equation (4) while the net force owing to viscous shear stresses is shown off by the 1st component on the right side. The net rate at which thermal energy escapes due to fluid motion is represented by the 2nd and 3rd terms on the left side of equation (5). The 1st component on the right side of equation (5) shows the net input of thermal energy owing to $y$-direction, while the 2 nd term represents the viscous dissipation that remains.

In this case, when the influence of surface tension is insignificant at a distance of $y=h$ from the surface, shear stresses operating in planes parallel to the fluid velocity are defined as

$$
\tau=\mu\left(\frac{\partial u}{\partial y}\right),
$$

where $\mu$ is a fluid property and is known as the dynamic viscosity and $\partial u / \partial y$ is the rate of shear stress.
The flow of heat at $y=h$ is defined by

$$
q=-k\left(\frac{\partial T}{\partial y}\right)
$$

In the above equation, the constant $k$ is called thermal conductivity. Equation (8) tells us that if temperature decreases with $y$ then $q$ will be positive and it will flow in the $y$ direction, if temperature increases with $y$ then $q$ will be negative and it will flow opposite in the $y$-direction.

2.2. Similarity Transformations. Here, we use the stream function $\psi(x, y, t)$ to specify the velocity terms:

$$
\begin{aligned}
& u=\frac{\partial \psi}{\partial y}=\left(\frac{b x}{1-\gamma t}\right) f^{\prime}(\eta), \\
& v=-\frac{\partial \psi}{\partial x}=-\left(\frac{\nu b}{1-\gamma t}\right)^{\frac{1}{2}} f(\eta),
\end{aligned}
$$

satisfying continuity equation (3). New dependent and independent variables, $f, \theta$, and $\eta$ are, respectively, introduced as 


$$
f(\eta)=\frac{\psi(x, y, t)}{(\nu b / 1-\gamma t)^{1 / 2} x}, \quad \theta(\eta)=\frac{T_{0}-T(x, y, t)}{T_{\mathrm{ref}}\left(b x^{2} / 2 \nu(1-\gamma t)^{-} 3 / 2\right)}, \quad \eta=\left(\frac{b}{\nu(1-\gamma t)}\right)^{1 / 2} y .
$$

Substitute the similarity transformation equations (9)-(11) into equations (4) and (5) to obtain a set of nonlinear ODEs in the form of

$$
\begin{aligned}
& f^{\prime \prime \prime}-\left(M+K_{1}\right) f^{\prime}+f f^{\prime \prime}-S\left(f^{\prime}+\frac{\eta}{2} f^{\prime \prime}\right)-(1+F)_{1} f^{\prime 2}=0 \\
& \left(1+\frac{4}{3} R\right) \theta^{\prime \prime}+E c \operatorname{Pr} f^{\prime \prime 2}-\operatorname{Pr}\left[\frac{S}{2}\left(3 \theta-\eta \theta^{\prime}\right)+2 \theta f^{\prime}-\theta^{\prime} f\right]=0 .
\end{aligned}
$$

Following are the corresponding boundary constraints of the differential equations:

$$
f(0)=0, f^{\prime}(0)=1, \theta(0)=1, f(\beta)=\frac{S \beta}{2}, \theta^{\prime}(\beta)=0, f^{\prime \prime}(\beta)=0,
$$

where the prime denotes derivative in terms of $\eta, S=\gamma / c$ is the unsteadiness parameter, $M=\sigma B^{2} / \rho c(1-\gamma t)$ is the magnetic parameter, $K_{1}=v \varepsilon^{2} / k c(1-\gamma t)$ is the porosity parameter, $E c=U^{2} / C_{p}\left(T_{s}-T_{0}\right)$ is the Eckert number, $\operatorname{Pr}=\mu C_{p} / k$ is the Prandtl number, and $R$ is thermal radiation. Despite the fact that the dimensionless film thickness $\beta$ is constant and only relies on $S$, the current film thickness $h$ is only depending on time $t$. So the free surface $\beta$ denotes the value of similarity variable by $\eta$ such that

$$
\beta=\left(\frac{c}{\nu(1-\gamma t)}\right)^{1 / 2} h
$$

By differentiating with respect to $t$, we have

$$
\frac{d h}{d t}=-\frac{\gamma \beta}{2}\left(\frac{c}{\nu}\right)^{-1 / 2}(1-\gamma t)^{-1 / 2}
$$

2.3. Physical Interest of the Problem. In this subsection, we calculated the skin friction coefficient $C_{f}$ and local Nusselt number $N u_{x}$ as

$$
C_{f}=\frac{-2 \tau_{s}}{\rho U^{2}}
$$

And

$$
N u_{x}=\frac{x}{k T_{r e f}}\left(q_{s}-q_{r}\right)
$$

From equations (7) and (8), at the surface $y=0$, the surface shear stress and surface heat flux are defined as

$$
\begin{aligned}
& \tau_{s}=\mu\left(\frac{\partial u}{\partial y}\right)_{y=0}, \\
& q_{s}=-k\left(\frac{\partial T}{\partial y}\right)_{y=0} .
\end{aligned}
$$

And the Rosseland estimation $q_{r}$ is given by [23]

$$
q_{r}=-\frac{16 \sigma^{*} T_{0}^{3}}{3 k^{*}}\left(\frac{\partial T}{\partial y}\right)_{y=0},
$$

where $\sigma^{*}$ is the Stefan-Boltzman constant and has the numerical value

$$
\sigma^{*}=5.670 \times 10^{-8} \mathrm{Wm}^{-2} \mathrm{~K}^{4}
$$

Inserting equations (19)-(21) into equations (17) and (18) to obtain $C_{f}$ and $N u_{x}$ in terms of $\mathrm{Re}_{x}$,

$$
C_{f}=-2 \operatorname{Re}_{x}^{-1 / 2} f^{\prime \prime}(0), N u_{x}=\left(1+\frac{4}{3} R\right) \frac{\operatorname{Re}_{x}^{3 / 2}}{2}(1-\gamma t)^{-1 / 2} \theta^{\prime}(0),
$$

where $\operatorname{Re}_{x}=c x^{2} / \nu(1-\gamma t)$ is the local Reynolds number and $R=4 \sigma^{*} T_{0}^{3} / k k^{*}$ is the radiation parameter.

\section{Solution by HAM}

HAM is applied for the solutions of equations (12) and (13) with boundary constraints (12). Mathematica is used for this purpose. The modeled equations are solved by HAM, the basic derivation is identified as 


$$
L_{\widehat{\theta}}(\widehat{\theta})=\widehat{\theta}^{\prime \prime}-\widehat{\theta}, L_{\widehat{f}}(\widehat{f})=\widehat{f}^{\prime \prime \prime}-\widehat{f}^{\prime}
$$

Linear operators $L_{\widehat{f}}$, and $L_{\widehat{\theta}}$ are specified by

$$
L_{\widehat{f}}\left(\gamma_{1}+\gamma_{2} e^{-\eta}+\gamma_{3} e^{\eta}\right)=0, L_{\widehat{\theta}}\left(\gamma_{4} e^{-\eta}+\gamma_{5} e^{\eta}\right)=0 .
$$
follows:

$$
\begin{array}{r}
N_{\widehat{f}}[\widehat{f}(\eta ; \zeta)]=\widehat{f}_{\eta \eta \eta}-S\left(\frac{1}{2} \eta \widehat{f}_{\eta \eta}+\widehat{f}_{\eta}\right)-\left(M+K_{1}\right) \widehat{f}_{\eta}-\left(1 F_{1}\right) \hat{f}_{\eta}^{2}+\hat{f} \hat{f}_{\eta \eta} \\
N_{\widehat{\theta}}[\widehat{\theta}(\eta ; \zeta), \hat{f}(\eta ; \zeta)]=\left(1+\frac{4}{3} R\right) \hat{\theta}_{\eta \eta}+E c \operatorname{Pr} \widehat{f}_{\eta \eta}^{2}+\operatorname{Pr}\left[\frac{S}{2}\left(\eta \widehat{\theta}_{\eta}+3 \hat{\theta}\right)+2 \widehat{\theta} \hat{f}_{\eta}-\hat{f}_{\eta}\right] .
\end{array}
$$

The $0^{\text {th }}$ order system for equations (12) and (13) is presented as

$$
\begin{array}{r}
(1-\zeta) L_{\widehat{f}}\left[\widehat{f}(\eta ; \zeta)-\widehat{f}_{0}(\eta)\right]=p \hbar_{\hat{f}} N_{\widehat{f}}[\widehat{f}(\eta ; \zeta)], \\
(1-\zeta) L_{\widehat{\theta}}\left[\widehat{\theta}(\eta ; \zeta)-\widehat{\theta}_{0}(\eta)\right]=p \hbar_{\hat{\theta}} N_{\widehat{\theta}}[\widehat{\theta}(\eta ; \zeta), \widehat{f}(\eta ; \zeta)],
\end{array}
$$

$$
\begin{aligned}
& \left.\hat{f}(\eta ; \zeta)\right|_{\eta=0}=0,\left.\frac{\partial \hat{f}(\eta ; \zeta)}{\partial \eta}\right|_{\eta=0}=1,\left.\frac{\partial^{2} \widehat{f}(\eta ; \zeta)}{\partial \eta^{2}}\right|_{\eta=\beta}=0,\left.\frac{\partial \hat{\theta}(\eta ; \zeta)}{\partial \eta}\right|_{\eta=\beta}=0 \\
& \left.\hat{f}(\eta ; \zeta)\right|_{\eta=\beta}=\frac{S \beta}{2},\left.\hat{\theta}(\eta ; \zeta)\right|_{\eta=0}=1 .
\end{aligned}
$$

$\hbar_{-}$and $\hbar_{-}$are utilized to standard for the solution confluence whereas the embedding constraint is $\zeta \in[0,1]$; when $\zeta=0$ and $\zeta=1$, we get the following:

$$
\widehat{\theta}(\eta ; 1)=\hat{\theta}(\eta), \hat{f}(\eta ; 1)=\widehat{f}(\eta) .
$$

Through Taylor's series, $\hat{f}(\eta ; \zeta)$ and $\hat{\theta}(\eta ; \zeta)$ are expanded for $\zeta=0$ :

$$
\widehat{\theta}(\eta ; \zeta)=\widehat{\theta}_{0}(\eta)+\sum_{n=1}^{\infty} \widehat{\theta}_{n}(\eta) \zeta^{n}, \widehat{f}(\eta ; \zeta)=\widehat{f}_{0}(\eta)+\sum_{n=1}^{\infty} \widehat{f}_{n}(\eta) \zeta^{n} \widehat{f}_{n}(\eta)=\left.\frac{1}{n !} \frac{\partial \widehat{f}(\eta ; \zeta)}{\partial \eta}\right|_{p=0}, \widehat{\theta}_{n}(\eta)=\left.\frac{1}{n !} \frac{\partial \widehat{\theta}(\eta ; \zeta)}{\partial \eta}\right|_{p=0}
$$

while BCs are

Now

$\widehat{f}^{\prime}(0)=1, \hat{f}(0)=0, \widehat{\theta}(0)=1, \hat{f}^{\prime \prime}(\beta)=0, \widehat{\theta}(\beta)=0, \widehat{f}(\beta)=\frac{S \beta}{2}$.

$$
\begin{aligned}
& \mathfrak{R}_{n}^{\widehat{f}}(\eta)=\widehat{f}_{n-1}^{\prime \prime}-S\left(\widehat{f}_{n-1}^{\prime}+\frac{1}{2} \eta \widehat{f}_{n-1}^{\prime \prime}\right)-\left(M+K_{1}\right) \hat{f}_{n-1}^{\prime}-(1-F)_{1} \widehat{f}_{n-1}^{\prime 2}+\sum_{j=0}^{w-1} \widehat{f}_{w-1-j} \widehat{f}_{j}^{\prime \prime} \\
& \mathfrak{R}_{n}^{\widehat{\theta}}(\eta)=\left(1+\frac{4}{3} R\right)\left(\widehat{\theta}_{n-1}^{\prime \prime}\right)+\operatorname{Pr} E c \widehat{f}_{n-1}^{\prime \prime 2}+\left[\frac{S}{2}\left(\eta \widehat{\theta}_{n-1}^{\prime}+3 \widehat{\theta}_{n-1}\right)-2 \sum_{j=0}^{w-1} \widehat{\theta}_{w-1-j} \widehat{f}_{j}^{\prime}-\sum_{j=0}^{w-1} \widehat{\theta}_{w-1-j}^{\prime} \widehat{f}_{j}\right],
\end{aligned}
$$




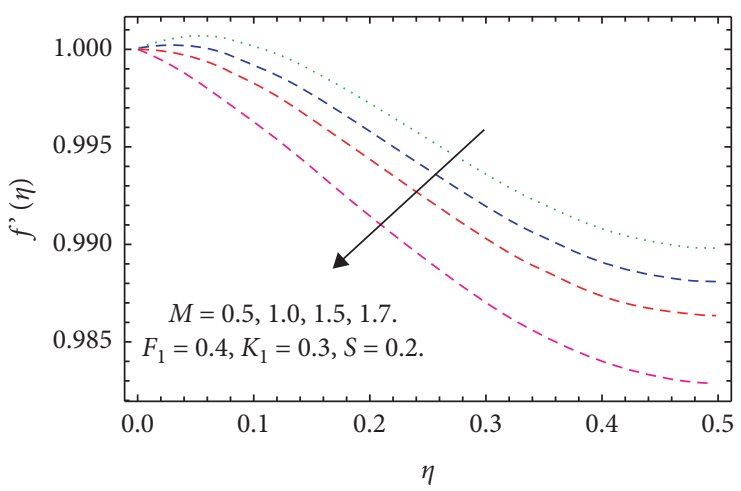

(a)

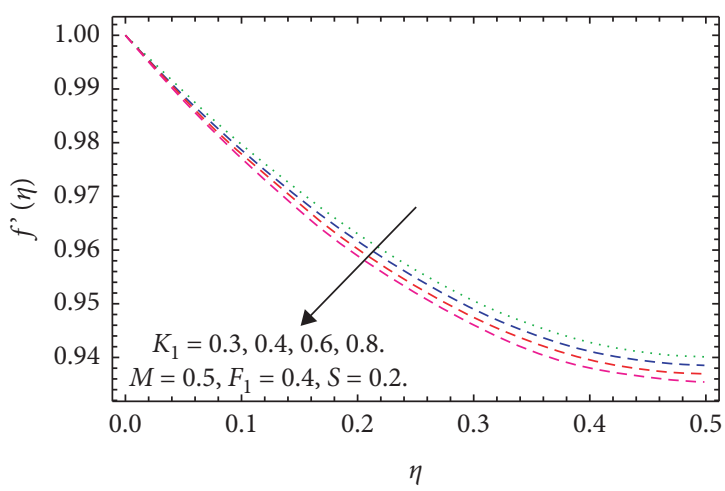

(b)

Figure 2: (a) Influence of $M$ on $f^{\prime}(\eta)$. (b) Influence of $K_{1}$ on $f^{\prime}(\eta)$.

while

$$
\chi_{n}=\left\{\begin{array}{lll}
0, & \text { if } & n \leq 1 \\
1, & \text { if } & n>1
\end{array}\right.
$$

\section{Result and Discussion}

The present work analyzes the Darcy-Forchheimer laminar thin film flow with MHD and heat transfer on an unsteady horizontal stretched surface in the absence of viscous dissipation, thermal radiation, and magnetic field of impact. The numerical, as well as analytical solutions, is given. Using similarity transformations, the governing partial differential equations of heat transfer flow are transformed into a nonlinear ordinary differential equation. Geometric representations of the problem are plotted in Figure 1 and the impact of physical parametric quantity on $f^{\prime}(\eta)$ and $\theta(\eta)$ curves are depicted in Figures 2-5. Figures 2 and 3 show the impact of $M, K_{1}, S$, and $F_{1}$ on $f^{\prime}(\eta)$. The velocity profile reduces when $(M)$ increases, as shown in Figure 2(a). This is due to the reducing nature of the Lorentz forces which reduces the motion of thin-film fluid in the boundary layer and raises its temperature. Figures 2(b) and 3(b) convey that the fluid velocity drop by rising values of $\left(K_{1}\right)$ and $\left(F_{1}\right)$. This is because the increasing hydromagnetic boundary layer increases the strength of porosity. Figure 3(a) illustrates the distribution of velocity gradient in the film decreases by rising values of unsteadiness parameter. As the value of $(S)$ in the system rises, the thickness of the boundary layer declines, preventing the establishment of a transition from laminar to turbulent flow. The effect of embedded parameters $\operatorname{Pr}, R, S$, and $E c$ on temperature profiles is exhibited in Figures 4 and 5. Figure 4(a) displays the variation of temperature reduces, as the Prandtl number increases. Being the ratio of molecular diffusivity of momentum to the molecular diffusivity of heat, it demonstrates the relative thickness of the momentum boundary layer to the thermal boundary layer. The rise in Prandtl number (Pr) decreases the temperature of the fluid. Increasing the Prandtl number, the thermal conductivity of the fluid decreases and the rate of heat transfer through stretch surface reduces; consequently, the temperature and thermal boundary layer reduce. Figure 4(b) demonstrates the temperature gradient for different values of $(R)$. As the values of $(R)$ upsurge, the temperature of thin film declines. Physically, when the radiation parameter increases, the Rosseland radiative absorption coefficient $k^{*}$ declines as defined by the expression for $R=4 \sigma^{*} T_{0}^{3} / k k^{*}$. Therefore, the heat flux diminishes. That is why the thin film temperature reduces. Figure 5(a) discusses the impact of unsteadiness parameter $(S)$ on temperature profiles. As the values of $(S)$ rise, the thermal boundary layer grows as a result, and the temperature field becomes increment for the nanofluid. Figure 5(b) demonstrates that increases in the value of $(E c)$ produce more temperature in the thin film. Eckert number is the ratio among kinetic energy and enthalpy, which is detected in the dissipation function of the energy equation, also known as the dissipation parameter. $E c=0$ means there is no viscous dissipation. Figure 5(b) displays that the behavior of $(E c)$ is directly proportional to the temperature, as $(E c)$ upsurge viscous dissipation rises, i.e., kinetic energy transformed into heat energy which in turn raises the temperature.

The physical significance of embedded parameters are displayed in Table 1 and the numerical values of skin friction and local Nusselt number are displayed in Tables 2 and 3. Table 2 demonstrates that the skin friction coefficient increases by rising various parameters, namely, $S, F_{1}, M$, and $K_{1}$. As these parameters increase, the unsteady parameter, the skin friction enhances due to the exertion of a drag force in the fluid thin film. The Lorentz force is a rising magnetic field that increases the resistive force on fluid movement. That is why more values of $(M)$ enhance $C_{f}$. Table 3 lists the numerical values of $N u_{x}$ for a few physical variables, that is, $E c, S, \operatorname{Pr}$, and $R$. The Eckert number is normally made up of the nanofluid thermal conductivity components, which is used to raise the temperature field, which has the reverse effect on cooling operations, and hence the Nusselt number reduced for high values of $(E c)$. With an increase in $(S)$, the boundary layer temperature of fluid raises, increasing the heat source of the thin film fluid; as a result, $N u_{x}$ boosts. In this table, we may observe that the Nusselt number upsurges as $(R)$ grows, but $N u_{x}$ drops as (Pr) grows. Table 4 displays excellent agreement between the HAM and ND solve solution through computer-based package Mathematica 11.1.0. 


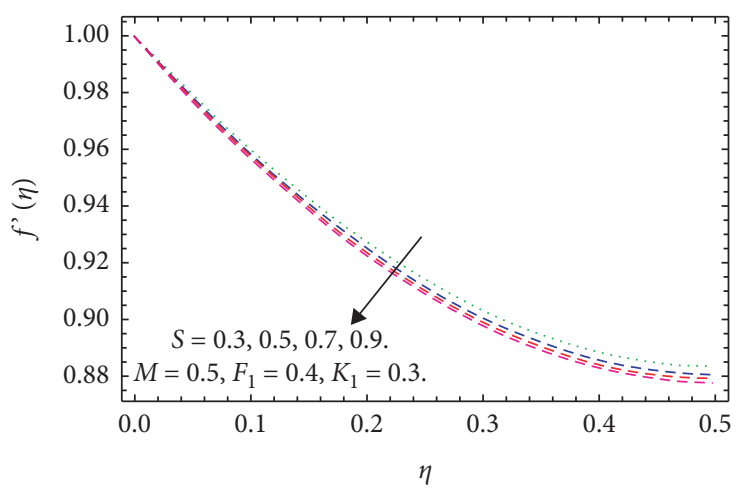

(a)

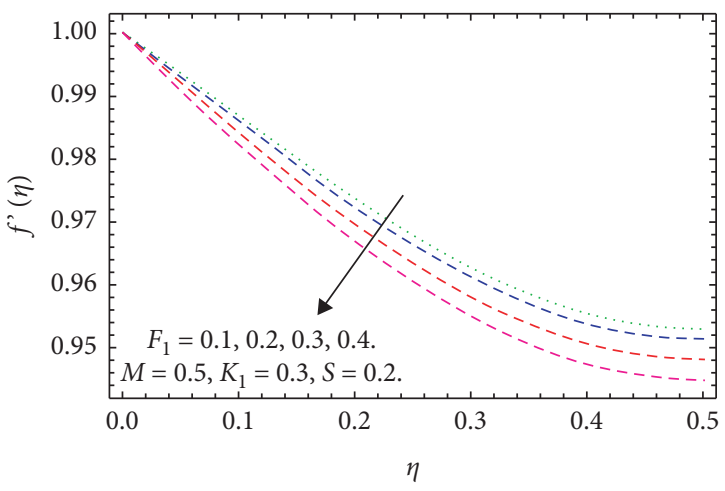

(b)

Figure 3: (a) Impact of $S$ on $f^{\prime}(\eta)$. (b) Impact of $F_{1}$ on $f^{\prime}(\eta)$.

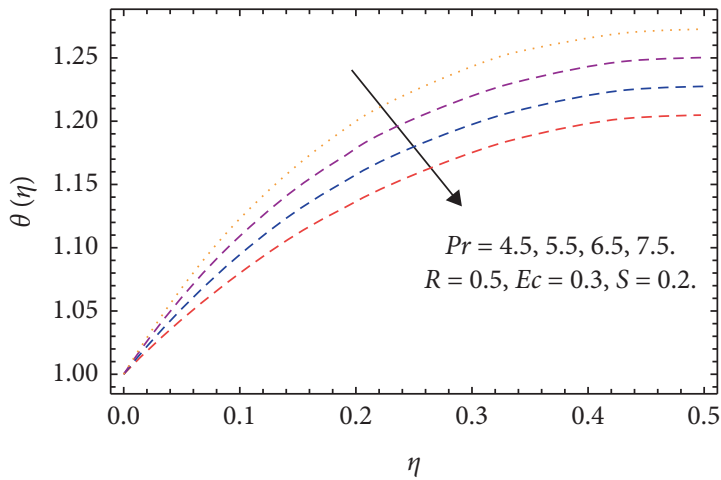

(a)

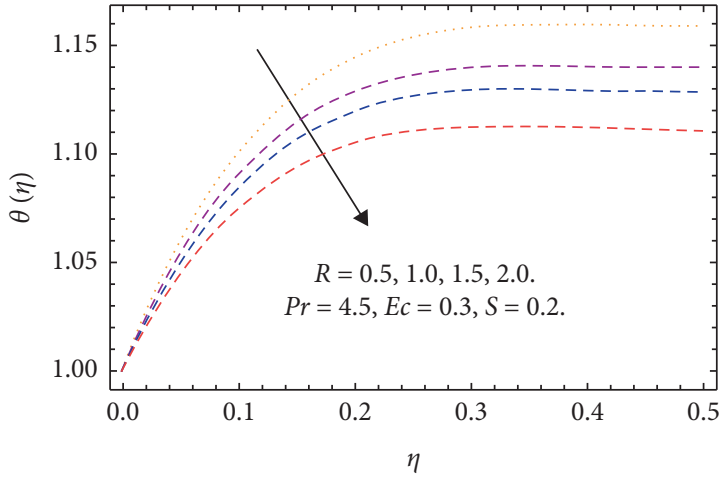

(b)

Figure 4: (a) Influence of $\operatorname{Pr}$ on $\theta(\eta)$. (b) Influence of $R$ on $\theta(\eta)$.

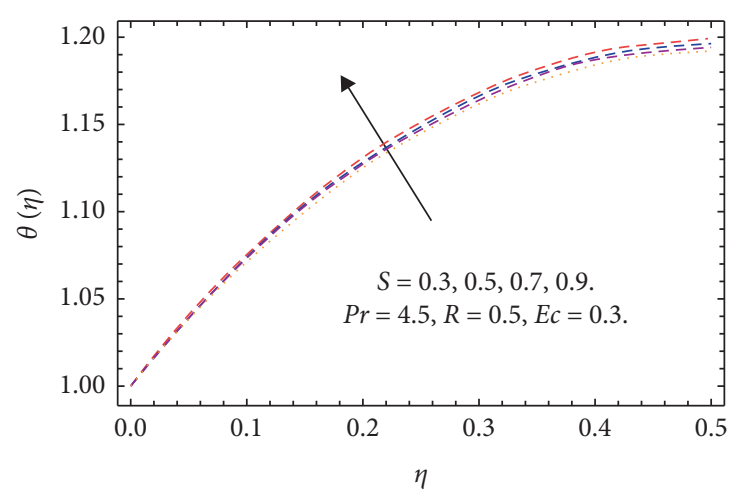

(a)

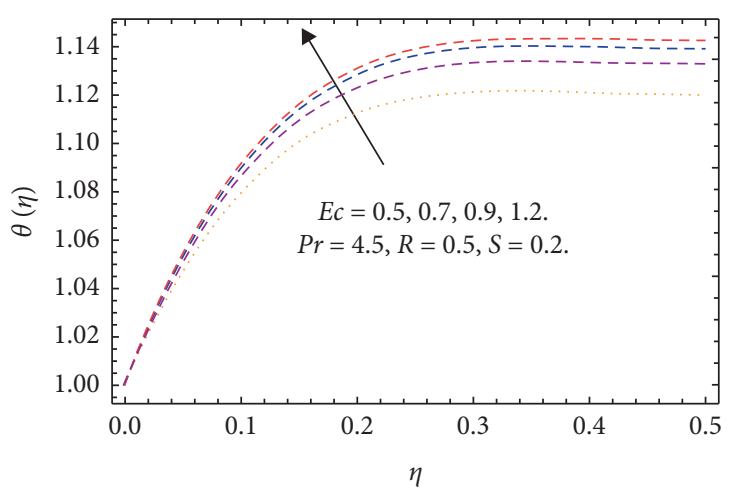

(b)

FIgURE 5: (a) Influence of $S$ on $\theta(\eta)$. (b) Influence of $E c$ on $\theta(\eta)$.

TABle 1: Dimensionless groups.

\begin{tabular}{lccc}
\hline Group & Symbol & Definition & Physical interpretation \\
\hline $\begin{array}{l}\text { Local Nusselt } \\
\text { number }\end{array}$ & $N u_{x}$ & $(1+4 / 3 R) \operatorname{Re}_{x}^{3 / 2} / 2(1-\gamma t)^{-1 / 2} \theta^{\prime}(0)$ & $\begin{array}{c}\text { Basic dimensionless convective heat transfer coefficient (ratio of } \\
\text { convective heat transfer to conduction in a fluid slab of thickness h) }\end{array}$ \\
$\begin{array}{l}\text { Local Reynolds } \\
\text { number }\end{array}$ & $R_{x}$ & $\rho U x / \mu$ & $\begin{array}{l}\text { The ratio of inertia to viscous forces } \\
\text { Eckert number }\end{array}$ \\
$\begin{array}{l}\text { Prandtl number } \\
\text { Skin friction }\end{array}$ & $\mathrm{Pr}$ & $\mathrm{C}_{f} / C_{p}\left(T_{s}-T_{0}\right)$ & $\begin{array}{c}\text { The kinetic energy of the flow relative to the boundary layer's enthalpy } \\
\text { difference }\end{array}$ \\
\hline
\end{tabular}


TABLE 2: Impact of different physical parameters on $C_{f}=-2\left[R e_{x}\right]^{-1 / 2} f^{\prime \prime}(0)$.

\begin{tabular}{ccccc}
\hline$S$ & $F_{1}$ & $M$ & $K_{1}$ & $-2\left[\mathrm{Re}_{x}\right]^{-1 / 2} f^{\prime \prime}(0)$ \\
\hline 0.3 & 0.3 & 0.4 & 0.3 & 2.86145012 \\
0.5 & & & 2.71436543 \\
0.7 & & & 2.01536798 \\
& 0.2 & & 1.83554023 \\
& 0.3 & & 1.73570141 \\
& 0.4 & & 1.49136523 \\
& & 0.4 & & 1.07413036 \\
& & 0.9 & 0.78652151 & 0.54037813 \\
& & 1.0 & 0.3 & 0.73156027 \\
& & & 0.4 & 0.47276531 \\
\hline
\end{tabular}

TABLE 3: Influence of various physical parameters over $N u_{x}=\delta y / \delta x\left[(1+4 / 3 R) R e_{x}^{3 / 2} / 2(1-\gamma t)^{-1 / 2} \theta^{\prime}(0)\right]$.

\begin{tabular}{ccccc}
\hline$E c$ & $S$ & $\operatorname{Pr}$ & $R$ & {$\left[(1+4 / 3 R) \operatorname{Re}_{x}^{3 / 2} / 2(1-\gamma t)^{-1 / 2} \theta^{\prime}(0)\right]$} \\
\hline 0.3 & 0.2 & 4.5 & 0.1 & 1.14202163 \\
0.5 & & & 1.07524301 \\
0.7 & & & 0.85345625 \\
& 0.2 & & 1.43180429 \\
& 0.3 & & 1.67459736 \\
& 0.7 & 4.5 & & 1.85128625 \\
& & 5.5 & 0.56262107 & 0.35129328 \\
& & & 0.16843568 \\
& & 0.5 & 1.5 & 1.43751020 \\
\end{tabular}

TABLE 4: Comparison of HAM and ND solve solution. $S=0.25, K_{1}=0.5, \beta=0.2, \operatorname{Pr}=6.4, M=0.5, R e_{x}=3.5, E c=0.8$.

\begin{tabular}{lcccccc}
\hline$\eta$ & & $f(\eta)$ & & $f^{\prime}(\eta)$ & & $\theta(\eta)$ \\
& HAM & ND solve & HAM & ND solve & HAM & ND solve \\
\hline 0 & 0 & 0 & 0.0457219 & 0.0457188 & 0.74 \\
0.5 & 0.05269587 & 0.05259644 & 0.06486865 & 0.06486847 & 0.60686886 & 0.60686799 \\
1.0 & 0.07173028 & 0.07172984 & 0.13425892 & 0.13425789 & 0.68623535 & 0.68623512 \\
1.5 & 0.16257245 & 0.16257196 & 0.13457543 & 0.13455484 & 0.74278208 & 0.74278193 \\
2.0 & 0.33723452 & 0.33713407 & 0.13273174 & 0.13272172 & 0.77686707 & 0.77685689 \\
2.5 & 0.27837542 & 0.27837478 & 0.12693225 & 0.12693218 & 1.03520089 & 1.03520056 \\
3.0 & 0.35155617 & 0.35145408 & 0.11862151 & 0.11862137 & 1.04484706 & 1.04484682 \\
3.5 & 0.36092614 & 0.36072302 & 0.05430493 & 0.05430466 & 1.05148879 & 1.05147865 \\
4.0 & 0.51720249 & 0.51706234 & 0.05587103 & 0.05587094 & 1.05852606 & 1.05852588 \\
4.5 & 0.52453042 & 0.52443205 & 0.05747184 & 0.05747172 & 1.06117499 & 1.06117458 \\
5.0 & 0.53436276 & 0.53426034 & 0.06316589 & 0.06316581 & 1.06254447 & 1.06254456 \\
5.5 & 0.54813433 & 0.54803512 & 0.06141789 & 0.06141749 & 1.04604842 & 1.04506683 \\
6.0 & 0.51242352 & 0.51242409 & 0.05967122 & 0.05968099 & 1.00678869 & 1.00648792 \\
6.5 & 0.54354575 & 0.54354514 & 0.05675423 & 0.05675401 & 0.95842587 & 0.95842484 \\
7.0 & 0.55501596 & 0.55501485 & 0.05264163 & 0.05264145 & 0.96204572 & 0.96204566 \\
7.5 & 0.55192046 & 0.55192013 & 0.05171947 & 0.05171824 & 0.92740813 & 0.92740799 \\
\hline
\end{tabular}




\section{Concluding Remarks}

The boundary layer two-dimensional Darcy-Forchheimer laminar thin film flow over a horizontal stretched sheet with the impact of thermal radiation and viscous dissipation are considered. The cause of heat source and thermal radiation over an unsteady stretched surface inserted in a porous medium in the absence of a magnetic field has been solved by HAM. The important key findings of this research work are given below:

(i) Velocity profile reduces for raise in values of $M$ and $K_{1}$

(ii) When the values of $S$ and $F_{1}$ increase, the velocity profile reduces

(iii) Temperature field increases with an increase in the values of $S$ and $E c$

(iv) With increasing values of $\operatorname{Pr}$ and $R$, the temperature field reduces

(v) Skin friction coefficient rises as upsurges the values of physical parameters that is $S, F_{1}, M$, and $K_{1}$

(vi) By increasing the values of $S$ and $R$, the local Nusselt number upsurge while it declines for more values of Ec and $\operatorname{Pr}$

\section{Nomenclature}

\begin{tabular}{|c|c|}
\hline Symbols: & Description \\
\hline$u:$ & Horizontal velocity component $\left(\mathrm{ms}^{-1}\right)$ \\
\hline$v:$ & Vertical velocity component $\left(\mathrm{ms}^{-1}\right)$ \\
\hline$x:$ & Horizontal coordinate $(\mathrm{m})$ \\
\hline$y:$ & Vertical coordinate $(\mathrm{m})$ \\
\hline$c:$ & Stretching rate $\left(\mathrm{s}^{-1}\right)$ \\
\hline$U:$ & Sheet velocity $\left(\mathrm{ms}^{-1}\right)$ \\
\hline$T:$ & Temperature $(\mathrm{K})$ \\
\hline$h:$ & Film thickness (m) \\
\hline$f:$ & $\begin{array}{l}\text { Dimensionless stream function, equation } \\
\text { (9) }\end{array}$ \\
\hline$C_{f}:$ & Skin friction coefficient, shown in Table 1 \\
\hline$t:$ & Time $(s)$ \\
\hline S: & Unsteadiness parameter, $\gamma / c$ \\
\hline$C_{p}:$ & Specific heat $\left(\mathrm{JKg}^{-1} \mathrm{~K}^{-1}\right)$ \\
\hline$q:$ & Heat flux, $-k(\partial T / \partial y)\left(\mathrm{Js}^{-1} \mathrm{~m}^{-1}\right)$ \\
\hline M: & Magnetic parameter, $\sigma B^{2} / \rho c(1-\gamma t)$ \\
\hline$K_{1}:$ & Porosity parameter, $\nu \varepsilon^{2} / k c(1-\gamma t)$ \\
\hline $\operatorname{Re}_{x}:$ & Local Reynolds number, shown in Table 1 \\
\hline Pr: & Prandtl number, shown in Table 1 \\
\hline$N u_{x}:$ & Local Nusselt number, shown in Table 1 \\
\hline$E c:$ & Eckert number, shown in Table 1 \\
\hline Greek & Descriptions: \\
\hline Symbols: & \\
\hline$\beta$ & $\begin{array}{l}\text { Dimensionless film thickness, } \\
(c / v(1-\gamma t))^{1 / 2} h\end{array}$ \\
\hline$\theta$ : & Dimensionless temperature, equation (9) \\
\hline$\gamma:$ & Constant $\left(\mathrm{s}^{-1}\right)$ \\
\hline$k$ : & Thermal conductivity $\left(\mathrm{m}^{2} \mathrm{~s}^{-1}\right)$ \\
\hline$\nu:$ & Kinematic viscosity $\left(\mathrm{m}^{2} \mathrm{~s}^{-1}\right)$ \\
\hline
\end{tabular}

$\begin{array}{ll}\mu: & \text { Dynamic viscosity }\left(\mathrm{Kgm}^{-1} \mathrm{~s}^{-1}\right) \\ \eta: & \text { Similarity variable, equation }(9) \\ \tau: & \text { Shear stress, } \mu(\partial u / \partial y)\left(\mathrm{Kgm}^{-1} \mathrm{~s}^{-2}\right) \\ \psi: & \text { Stream function }\left(\mathrm{m}^{2} \mathrm{~s}^{-1}\right) \\ \rho: & \text { Density }\left(\mathrm{Kgm}^{-3}\right)\end{array}$

Subscripts: Descriptions:

o: $\quad$ Origin

Ref: $\quad$ Reference value

$s: \quad$ Sheet

$x$ : $\quad$ Localized value

Superscripts: Descriptions:

I: $\quad$ lnd derivative

II: $\quad$ 2nd derivative

II!: $\quad 3 r d$ derivative

\section{Data Availability}

No data are available regarding this manuscript.

\section{Conflicts of Interest}

The authors declare that they have no conflicts of interest.

\section{References}

[1] R. Jan, H. Khan, P. Kumam, F. Tchier, R. Shah, and H. Bin Jebreen, "The Investigation of the Fractional-View Dynamics of Helmholtz Equations Within Caputo Operator," Computers, Materials \& Continua, vol. 68, no. 3, pp. 3185-3201, 2021.

[2] S. Khattak, I. Hussain, J. F. Gomez-Aguilar, and R. Jan, "Analysis of PD-type iterative learning control for discretetime singular system," Mathematical Methods in the Applied Sciences, pp. 1-14, 2021.

[3] H. M. Srivastava, R. Jan, A. Jan, W. Deebani, and M. Shutaywi, "Fractional-calculus analysis of the transmission dynamics of the dengue infection," Chaos: An Interdisciplinary Journal of Nonlinear Science, vol. 31, no. 5, Article ID 053130, 2021.

[4] F. M. Alharbi, M. Naeem, M. Zubair, M. Jawad, W. U. Jan, and R. Jan, "Bioconvection Due to Gyrotactic Microorganisms in Couple Stress Hybrid Nanofluid Laminar Mixed Convection Incompressible Flow with Magnetic Nanoparticles and Chemical Reaction as Carrier for Targeted Drug Delivery through Porous Stretching Sheet," Molecules, vol. 26, no. 13, p. 3954, 2021.

[5] C. Y. Wang, "Liquid film on an unsteady stretching surface," Quarterly of Applied Mathematics, vol. 48, no. 4, pp. 601-610, 1990.

[6] C. Wang, "Analytic solutions for a liquid film on an unsteady stretching surface," Heat and Mass Transfer, vol. 42, no. 8, pp. 759-766, 2006.

[7] M. Sufian, A. Mehmood, and A. Ali, "Time-dependent flow and heat transfer over a stretching cylinder," Chinese Journal of physics, vol. 50, no. 5, pp. 828-848, 2012.

[8] Y. Zhang, M. Zhang, and S. Qi, "Heat and Mass Transfer in a thin liquid film over an unsteable stretched sheet in the absence of variable magnetic field and thermosolutal capillarity," Mathematical Problems in Engineering, vol. 2016, Article ID 8521580, 2016. 
[9] K. Kalyani, K. Sreelakshmi, and G. Sarojamma, "The threedimensional flow of a non-Newtonian fluid over a stretching flat surface through a porous medium with surface convective conditions," Global Journal of Pure and Applied Mathematics, vol. 13, no. 6, pp. 2193-2211, 2017.

[10] K. Kalyani, K. Sreelakshmi, and G. Sarojamma, "Effect of thermal radiation on the Casson thin liquid film flow over a stretching sheet," Global Journal of Pure and Applied Mathematics, vol. 13, no. 6, pp. 1575-1592, 2017.

[11] C. D. S. Devi, H. S. Takhar, and G. Nath, "Unsteady mixed convection flow in stagnation region adjacent to a vertical surface," Wärme-Und Stoffübertragung, vol. 26, no. 2, pp. 71-79, 1991.

[12] A. K. Jhankal, R. N. Jat, and D. Kumar, "Magnetohydrodynamics (MHD) Forced Convective Flow and Heat Transfer Over a Porous Plate in a Darcy-Forchheimer Porous Medium in Presence of Radiation," International Journal of Current Research, vol. 9, no. 11, pp. 1663-1674, 2017.

[13] S. E. Waheed, "Flow and heat transfer in a Maxwell liquid film over an unsteady stretching sheet in a porous medium with radiation," SpringerPlus, vol. 5, no. 1, pp. 1061-14, 2016.

[14] I. Ahmad, M. Sajid, W. Awan et al., "MHD flow of a viscous fluid over an exponentially stretched surface in a porous medium," Journal of Applied Mathematics, vol. 2014, Article ID 256761, 2014.

[15] E. M. Sparrow and J. L. Gregg, "A boundary-layer treatment of laminar-film condensation," Journal of Heat Transfer, vol. 81, no. 1, pp. 13-18, 1959.

[16] B. S. Dandapat and P. C. Ray, "Film cooling on a rotating disk," International journal of non-linear mechanics, vol. 25, no. 5, pp. 569-582, 1990.

[17] B. S. Dandapat and P. C. Ray, "The effect of thermocapillarity on the flow of a thin liquid film on a rotating disc," Journal of Physics D: Applied Physics, vol. 27, no. 10, pp. 2041-2045, 1994.

[18] M. Kumari and G. Nath, "Unsteady MHD film flow over a rotating infinite disk," International journal of engineering science, vol. 42, no. 11-12, pp. 1099-1117, 2004.

[19] N. A. A. Mohd Nasir, N. A. Azeany, A. Ishak, and I. Pop, "Magnetohydrodynamic Flow and Heat Transfer Induced by a Shrinking Sheet," Mathematics, vol. 8, no. 7, p. 1175, 2020.

[20] H. N. Zaidi, M. Yousif, and S. Nazia Nasreen, "Effects of Thermal Radiation, Heat Generation, and Induced Magnetic Field on Hydromagnetic Free Convection Flow of Couple Stress Fluid in an Isoflux-Isothermal Vertical Channel," Journal of Applied Mathematics, vol. 2020, pp. 1-12, 2020.

[21] P. M. Kishore, V. Rajesh, and V. Verma, "The effects of thermal radiation and viscous dissipation on MHD heat and mass diffusion flow past an oscillating vertical plate embedded in a porous medium with variable surface conditions," Theoretical and Applied Mechanics, vol. 39, no. 2, pp. 99-125, 2012.

[22] E. Elbashbeshy and T. G. Emam, "Effects of thermal radiation and heat transfer over an unsteady stretching surface embedded in a porous medium in the presence of heat source or sink," Thermal science, vol. 15, no. 2, pp. 477-485, 2011.

[23] A. S. Khan, Y. Nie, and Z. Shah, "Impact of thermal radiation on magnetohydrodynamic unsteady thin film flow of Sisko fluid over a stretching surface," Processes, vol. 7, no. 6, p. 369, 2019.

[24] S. Palani, B. R. Kumar, and P. K. Kameswaran, "Unsteady MHD flow of an UCM fluid over a stretching surface with higher order chemical reaction," Ain Shams Engineering Journal, vol. 7, no. 1, pp. 399-408, 2016.
[25] M. S. Abel, N. Mahesha, and J. Tawade, "Heat transfer in a liquid film over an unsteady stretching surface with viscous dissipation in presence of external magnetic field," Applied Mathematical Modelling, vol. 33, no. 8, pp. 3430-3441, 2009.

[26] S. Shateyi and J. Prakash, "A new numerical approach for MHD laminar boundary layer flow and heat transfer of nanofluids over a moving surface in the presence of thermal radiation," Boundary value problems, vol. 2014, no. 1, pp. 1-12, 2014.

[27] F. Mabood, W. A. Khan, and A. I. M. Ismail, "MHD boundary layer flow and heat transfer of nanofluids over a nonlinear stretching sheet: a numerical study," Journal of Magnetism and Magnetic Materials, vol. 374, pp. 569-576, 2015. 